\title{
Comprehensive analysis of diverse ribonucleoprotein complexes
}

\author{
Marlene Oeffinger ${ }^{1}$, Karen E Wei ${ }^{1}$, Richard Rogers ${ }^{2}$, Jeffrey A DeGrasse ${ }^{1}$, Brian T Chait ${ }^{1}$, John D Aitchison ${ }^{2}$ \& \\ Michael P Rout ${ }^{1}$
}

\begin{abstract}
The study of the dynamic interactome of cellular ribonucleoprotein (RNP) particles has been hampered by severe methodological limitations. In particular, the affinity purification of intact RNP complexes from cell lysates suffers from RNA degradation, loss of interacting macromolecules and poor overall yields. Here we describe a rapid affinity-purification method for efficient isolation of the subcomplexes that dynamically organize different RNP biogenesis pathways in Saccharomyces cerevisiae. Our method overcomes many of the previous limitations to produce large RNP interactomes with almost no contamination.
\end{abstract}

During and after eukaryotic transcription, RNA is packaged into RNP complexes, then modified, spliced, folded and exported into the cytoplasm through nuclear pore complexes (NPCs). There are two main forms of RNPs. Each contains a different type of RNA: mRNPs contain mRNA, and rRNPs contain ribosomal RNA (rRNA). Each also uses discrete processing factors, which associate with RNPs in a dynamic fashion to define the order of transcript maturation. The function and composition of many of these RNP complexes, however, is poorly understood.

In recent years, there have been considerable efforts to study the composition of mRNPs and rRNPs in greater detail. A favored approach has been to purify these complexes from cell lysates ${ }^{1-4}$. But the limitations of presently available purification techniques pose a major hurdle to the study of RNA processing and export. Here we describe a method that aims to overcome these limitations and capture the dynamic interactome of maturing RNP complexes. We used this method to analyze complexes along both the ribosome and mRNA biogenesis pathways.

\section{RESULTS}

\section{A strategy for isolation of RNP complexes}

Our strategy comprises seven steps that we optimized for efficient isolation of intact RNP complexes (Fig. 1 and Supplementary Methods online). Collected cells are rapidly frozen in liquid nitrogen and broken open in the solid phase by milling ${ }^{5}$, using a planetary ball mill that produces particles of $\sim 1-2 \mu \mathrm{m}$ in diameter (small enough for rapid and efficient RNP extraction). Because cell breakage occurs in the frozen state, we avoid damage to RNP complexes by released nucleases and proteases, as well as redistribution of proteins within the extract. The resulting frozen grindate is rapidly thawed into an RNP-compatible buffer (see Supplementary Methods), to produce an extract that is then immediately clarified by filtration. We isolate the tagged complexes using antibody-conjugated magnetic beads, rather than more commonly used antibody-conjugated resins such as Sepharose. The magnetic beads are small $(2.8 \mu \mathrm{m})$ and so have a large surface area-to-volume ratio that increases the speed of binding. Moreover, the beads are impermeable, such that the antibodies are densely conjugated to their exposed surface; hence, there is no theoretical upper limit to the size of isolated complexes (unlike with permeable resins such as Sepharose, which are limited by their pore size). Incubation times of $<30 \mathrm{~min}$ proved sufficient to recover $>90 \%$ of the tagged complex in every case studied.

\section{Clean isolation of RNP interactomes}

After elution, we subjected the isolated RNP complexes to parallel analyses of their proteome and their RNA. In all cases we recovered rich interactomes, with little evidence of nonspecific interactions. As an example, we isolated tagged Cbp80p, a component of the cap-binding complex (CBC) associated with the $5^{\prime}$ end of mRNAs ${ }^{6}$. We identified 51 known mRNA-associated proteins in this complex. Of these, 30 have previously been shown to associate with Cbp80p-containing complexes ${ }^{1,3,7}$. Many of these 51 components have been characterized as dynamically associating early mRNA processing and splicing factors (Supplementary Table 1 online). Because the $\mathrm{CBC}$ remains associated with the mRNA until it is exported, we also found components that are involved in later stages of mRNA maturation, including mRNA export factors and components of the $3^{\prime}$-end maturation machinery. We compared our approach to that of the tandem affinity purification (TAP) method $^{1}$, which recovered only three specific components of the CBC interactome: Cbp80p itself, Cbp20p and Kap60p (ref. 1). This comparison illustrates the far richer interactomes that can potentially be recovered with the present method.

The isolated RNP complexes are also virtually devoid of contaminants. We found no contaminants in our negative control and only one potential contaminant in the Cbp80p complex

${ }^{1}$ Rockefeller University, 1230 York Avenue, New York, New York 10021, USA. ${ }^{2}$ Institute for Systems Biology, 1141 N. 34th St., Seattle, Washington 98103, USA. Correspondence should be addressed to M.P.R. (rout@rockefeller.edu).

RECEIVED 23 JULY; ACCEPTED 12 SEPTEMBER; PUBLISHED ONLINE 7 OCTOBER 2007; DOI:10.1038/NMETH1101 
a

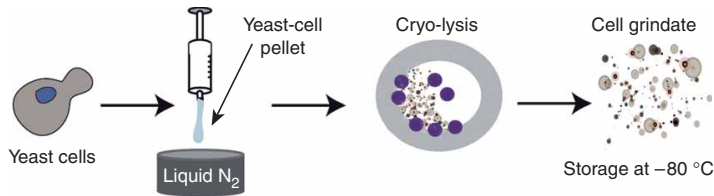

b

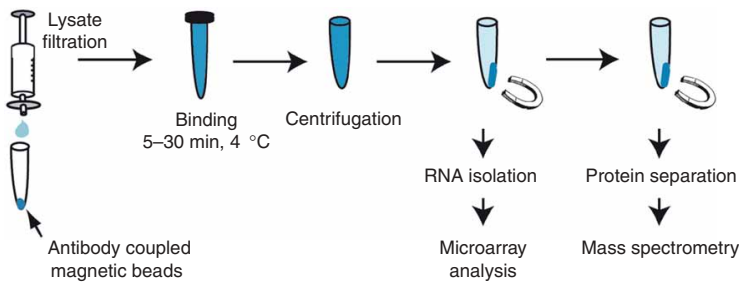

Figure 1 | Schematic outline of the single-step purification technique.

(a) Yeast cells are collected, lysed by cryo-milling and stored at $-80{ }^{\circ} \mathrm{C}$.

(b) The grindate is mixed with an RNP-compatible buffer, filtered and after a short incubation with antibody-conjugated magnetic beads, the complexes are isolated on a magnet, washed, eluted under denaturing conditions and their protein and RNA components are analyzed.

isolation. Indeed, we observed only three likely contaminants out of a total of 258 different proteins identified in all of the pullouts conducted for this study. For comparison, a previous study ${ }^{3}$ lists 66 common contaminants found in TAP pullouts, of which 15 had been detected in at least $10 \%$ of the isolations and 17 in the negative controls.

\section{Efficient isolation of diverse RNP complexes}

We illustrate the versatility of our approach by isolating two different types of RNP complexes (Supplementary Fig. 1a online): pre-ribosomes, using a tagged version of the pre-rRNA processing factor Nop15p (ref. 8), and exporting mRNPs, using tagged versions of the nuclear mRNA export factor Mex67p (ref. 9). As a control, we isolated Protein A ( $P r A)$ alone from a strain expressing just the PrA tag (Fig. 2a).

The RNA-binding protein Nop15p is associated with $90 \mathrm{~S}$ and pre-60S pre-ribosomal particles ${ }^{8}$. Nop15p has previously been used as a TAP-tagged bait protein in high-throughput proteomic studies, which identified 35 associated proteins, predominantly preribosomal processing factors ${ }^{3,7}$. We identified 136 proteins, including all of the previously identified proteins.

Of the identified proteins, 44 were ribosomal proteins and 89 were pre-ribosomal processing factors of $90 \mathrm{~S}$ and pre-60S particles (Fig. 2b). A problem that has plagued the field is cross-contamination of pre-40S components into pre-60S particles $3,7,10$.

\footnotetext{
Figure 2 | Isolation of PrA-tagged Nop15p complexes. (a) PrA was expressed under the control of the endogenous ZPR1 promoter and affinity purified using immunoglobulin gamma (IgG)-conjugated magnetic beads. (b) Nop15passociated complexes were affinity purified via the GFP or the PrA tag, and associated proteins were resolved by SDS-PAGE and visualized by staining with Coomassie blue. Proteins identified by mass spectrometry are listed on the right and in Supplementary Table 1. *, newly identified proteins; r-proteins, ribosomal proteins.
}

Notably, we found no pre-40S subunit components, other than those known to assemble onto the $90 \mathrm{~S}$ particle ${ }^{11}$. The identification of several exonucleases known to be involved in rRNA processing also demonstrates that the method we describe can be used to capture transiently interacting components (M.O., unpublished data $)^{12}$. Notably, a role for Nop15p in the control of cytokinesis has been suggested ${ }^{8}$ and is supported by the identification of three Nop15p-associated proteins with known roles in cytokinesis (Supplementary Table 2 online). All other co-isolating proteins are known to be part of the 90S and early pre-60S particles from different affinity-purification studies but have not previously been found together in the same affinity-purified isolate.

Although Mex67p has been extensively studied, it has proven difficult to identify its interaction partners. To date, affinity purification of tagged Mex67p has identified only two associated proteins ${ }^{13}$. By contrast, we identified 34 associated proteins in our purified Mex67p-PrA RNP complexes (Supplementary Fig. 1a). Consistent with previous studies and in accordance with the function of Mex67p in transporting mRNAs across the NPC, 23 of these proteins are NPC components (Supplementary Table 2). As also expected of an RNA export complex, we found the CBC, several known mRNA-associated proteins and karyopherins (Supplementary Fig. 1a); the association of only five of these proteins with Mex67p has previously been demonstrated (Supplementary Table 2).

The efficiency of our method does not depend on a particular protein tag, as we can isolate essentially identical interactomes using either a PrA or GFP tag on a given protein (Fig. 2b and Supplementary Fig. 1a). This method should thus be readily adaptable to other appropriately tagged constructs.

\section{High yields ensure recovery of a representative RNP pool}

It is imperative to obtain high yields during affinity purifications to ensure recovery of RNPs that are sufficiently representative of those associated with the bait protein in vivo ${ }^{14}$. Quantitative western blot analyses showed that we were able to isolate $\sim 95 \%$ of the tagged protein for Mex67p-PrA and $\sim 90 \%$ for Nop15p-PrA. In all the recovered complexes, the co-isolating proteins appear to be in nearstoichiometric amounts, as their Coomassie blue-stained bands are usually of similar intensities to that of the bait protein. 
a

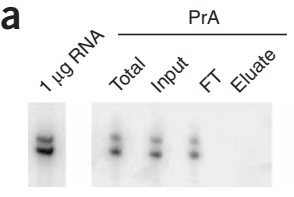

$\%$

$\begin{array}{llll}100 & 95 & 94 & 0.4\end{array}$
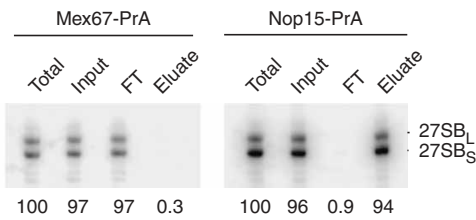

b

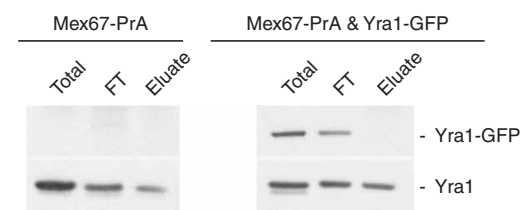

Figure 3 | Quantitation of recovered RNA and protein-rearrangement analysis during complex isolation. (a) Recovery of 27SB pre-rRNA with PrA alone, Mex67p-PrA and Nop15p-PrA was tracked throughout the affinity-purification procedure by primer extension of co-isolated RNA. One-twelfth of total sample was loaded in each lane; $1 \mu \mathrm{g}$ of cellular RNA was run for comparison. Total, total cell extract; input, cell extract after filtration; FT, immunologically depleted cell extract; eluate, RNA isolated by Proteinase K digest and phenolchloroform extraction. Samples were separated on a $6 \%$ polyacrylamide/urea gel. Percentage of input RNA recovered in each fraction is listed below each lane. (b) Affinity purification was carried out from either Mex67p-PrA grindate alone or mixed Mex67p-PrA/Yra1p-GFP grindate. Western blot analysis using anti-Yra1p (1:2,000) and anti-GFP (1:2,000). A 1/400 th of total sample was loaded in each lane for total, FT and eluate.

For quantitation of RNA recoveries, we determined yields by primer extension analysis of specific RNAs known to be associated with either Nop15p or Mex67p (refs. 8,15). To monitor recoveries of Nop15p-associated RNAs, we followed the ribosomal pre-RNA $27 \mathrm{SB}$, which is the precursor to mature $5.8 \mathrm{~S}$ and $25 \mathrm{~S}$ rRNAs and a component of pre-60S particles. PrA-tagged Nop15p purified $\sim 94 \%$ of the ribosomal precursor RNA 27SB from the lysate ${ }^{16}$ (Fig. 3a). Mex67p associates in the nucleus with maturing mRNAs before their export and is removed from mature mRNAs in the cytoplasm $^{9,17}$. A recent study has shown that $\sim 70 \%$ of mRNA within a yeast cell is polysome-associated, with $\sim 10 \%$ suggested to be nuclear mRNAs and the rest being free cytoplasmic mRNAs waiting to be either translated or degraded ${ }^{18}$. Consistent with this study, we found that Mex67p-PrA co-isolated with only a portion ( $\sim 29 \%$ ) of the total cellular PGK1 mRNA (Supplementary Fig. 1b). This portion is more than the amount of mRNA believed to be in the nucleus, raising the possibility that newly exported mRNAs may stay associated with a Mex67p-containing complex until they are bound to ribosomes for translation (to prevent them from being targeted by the degradation machinery).

\section{RNP complexes do not rearrange during isolation}

For the isolated RNP pool to represent the in vivo situation, we must ensure that RNP proteins do not rearrange on RNAs during the isolation procedure. To test for such rearrangements, we performed mixing experiments with grindates from cells expressing PrA-tagged Mex67p and cells expressing GFP-tagged Yralp. Yralp and Mex67p can be part of separate complexes, but are also found together in the same RNP particles immediately before export, such that Yralp and Mex67p partially copurify. We affinity purified Mex67p-associated complexes from Mex67p-PrA grindate mixed with equal amounts of Yralp-GFP grindate. Mex67p-PrA should copurify with the untagged form of Yralp with which it associated in vivo, but should any redistribution of Yralp occur during isolation, then both GFP-tagged and untagged Yralp would also copurify with Mex67p-PrA. We found that only untagged Yralp was present in the isolated Mex67p-PrA complexes (Fig. 3b). This demonstrates that no Yralp rearrangements took place during the isolation of Mex67p-associated RNPs and, by implication, that rearrangement between RNAs and their binding proteins is not a problem during our procedures.

We also used primer extension analyses to check for crosscontamination of RNAs between complexes during the isolation procedure, asking whether rRNAs are found in an mRNP complex isolation and vice versa. We did not detect any PGK1 mRNA associated with Nop15p-PrA (Supplementary Fig. 1b) or 27SB pre-ribosomal RNA with Mex67p (Fig. 3a), and PrA expressed alone did not purify either RNA, demonstrating the high level of purity and lack of RNA cross-contamination in our RNP isolates, that is, that the RNAs do not rearrange notably between RNP complexes during the isolation procedure.

\section{A global investigation of the mRNA maturation pathway}

The dynamics of mRNPs during different stages of mRNA maturation is still not well understood ${ }^{19}$. To demonstrate the efficacy and discovery potential of our purification method to a biologically challenging problem, we examined six known mRNP components for affinity purification, found in early, middle and late stages of the mRNA maturation pathway (Fig. 4).

The first component, Thp2p, is part of the THO complex, which forms co-transcriptionally on nascent transcripts ${ }^{20}$. Of the 15 proteins we isolated with tagged Thp2p (Supplementary Table 1), six had not been found in previous studies, including two known mRNA-associated factors (Yra2p and Npl3p) $)^{19}$, a histone, the $\mathrm{CBC}$ that associates with the mRNA $5^{\prime}$ end cotranscriptionally (Fig. 4), and a previously uncharacterized protein, Yhr127p (also found associating with Mex67p; Supplementary Fig. 1a). Notably, Yhr127p contains a canonical RNA-binding domain and specifically associates with several of the complexes in the mRNA maturation pathway (Fig. 4); we are now investigating its function. Again, with the exception of Vps1p, no common contaminants were present, and all the proteins found are known to participate in the mRNA maturation pathway. Likewise, in all the other five complex isolations discussed below, we found virtually no contaminants and many known mRNA-interacting factors.

The second component, Yralp, also associates early with the nascent mRNA and stays associated with mRNPs until they are exported through the NPC and reach the cytoplasm ${ }^{21,22}$. YralpPrA copurified with a total of 47 proteins, 25 of which are known mRNA-associated factors (Fig. 4). We also identified Mlp1p and Mlp2p, proteins associated with the NPC and the nuclear periphery, whose interaction with Yralp had previously been demonstrated ${ }^{23}$, and 24 proteins that had not been seen in any previous affinity purification using tagged Yralp, including NPC components, splicing factors, a protein (Esclp) found at the nuclear periphery and resembling in structure Mlp1p and Mlp2p (ref. 24), an E3 ubiquitin ligase (Tom1p) that functions during mRNA maturation ${ }^{25}$, and several mRNA-associated proteins. We also found that Yralp co-isolated with all of our purified mRNP complexes except the She $2 p$ complexes, affirming its presence from early to late stages of mRNA maturation ${ }^{21,22}$.

The third component, Sac3p, is an NPC-associated protein involved in mRNA export ${ }^{26}$. The fact that Sac3p is present in 
cells in very low copy numbers ${ }^{27}$ has made it a difficult candidate for affinity purification, and thus only 13 Sac3p-associating proteins have been identified to date by various methods (none using Sac3p affinity purifications). We, however, isolated and identified 45 Sac3p-associated proteins (Fig. 4); these include 22 nucleoporins, the endonuclease Yshlp involved in $3^{\prime}$-end maturation and many known mRNA-associated factors. We discussed the fourth and fifth components, Cbp80p and Mex67p, above (Fig. 4 and Supplementary Fig. 1a).

The sixth component, the RNA-binding protein She2p, has been extensively studied by both proteomic and RNomic approaches ${ }^{28-30}$. She2p is involved in the localization of ASH1 mRNA to the distal tip of daughter cells during anaphase ${ }^{28}$, which is accomplished by She 2 p binding directly to the RNA and engaging it via the linker protein She3p to the myosin Myo4p that then transports the complex along actin cables to the bud tip ${ }^{29}$. Using PrA-tagged She2p, our proteomic analysis identified the expected proteins She3p and Myo4p; in addition we found two other proteins, Myo2p and Act1p (Fig. 4). Myo2p, like Myo4p, is a type $\mathrm{V}$ myosin involved in actin-based transport of cargo $^{31}$, and
Actlp forms the actin cables along which mRNAs are transported ${ }^{32}$. Therefore, the two newly identified components fit the known function of the She $2 p$ complex.

The complexes isolated with the tagged mRNP proteins were discrete but showed clear compositional overlaps, reflecting that we are isolating different pools of RNPs along the mRNA maturation pathway (Fig. 4). Thp2p is the first of the tagged bait proteins to associate with mRNPs, and consistent with this, the complex contains the $\mathrm{CBC}$ as well as co-transcriptionally associated factors but no components of the NPC or the mRNA export machinery ${ }^{19}$. The Yralp-PrA associated complexes represent early, intermediate and exporting complexes, containing both the THO complex and the export machinery, as well as splicing and $3^{\prime}$-end maturation factors and proteins associated with the nuclear periphery, where exporting mRNPs first dock to the NPC. The compositions of both Mex67p- and Sac3p-associated complexes mirror their late role in mRNA maturation, being detected as associated immediately before and during mRNA export ${ }^{19}$. These latter complexes contain predominately nucleoporins, the $\mathrm{CBC}$ and other proteins known to accompany the mRNA through the NPC to the cytoplasm. The
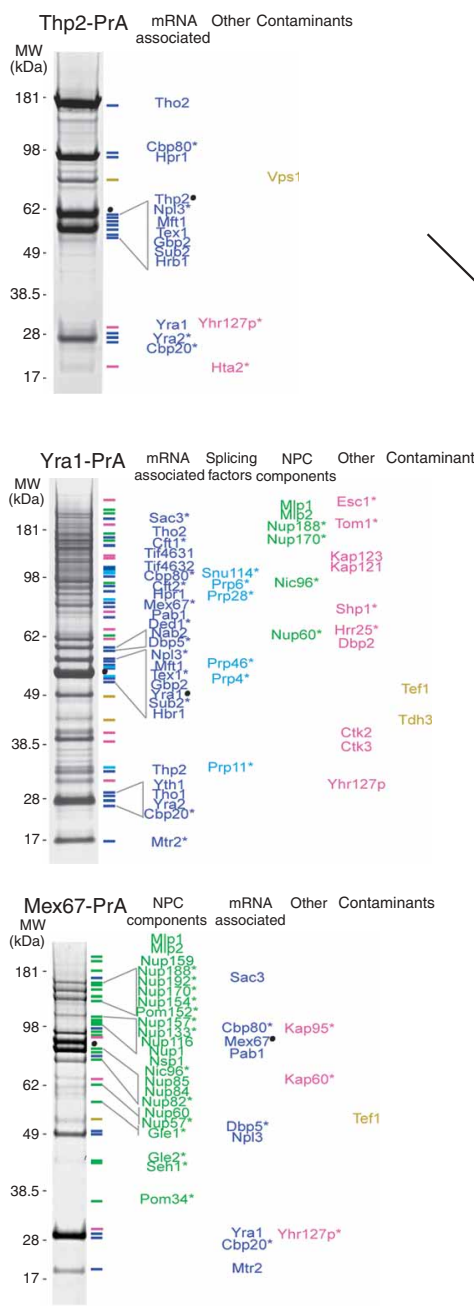
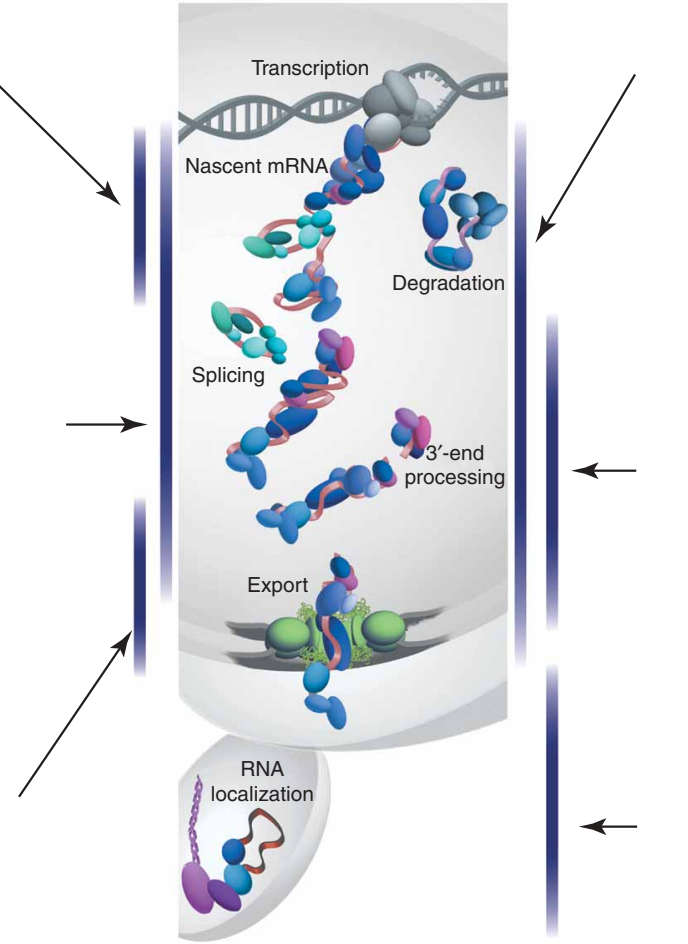

Figure 4 Analysis of specific complexes along the mRNA maturation pathway. Complexes associated with the indicated PrA-tagged proteins were affinity purified via the tag, resolved by SDS-PAGE and visualized by staining with Coomassie blue. Proteins identified by mass spectrometry are listed (also see Supplementary Table 1). Newly identified proteins are indicated by asterisks. Bars to the left and right of the diagram indicate the correlation of isolated complexes to stages of the mRNA maturation pathway. The complexes isolated with the tagged proteins show clear compositional overlaps and reflect different pools of RNPs along the mRNA pathway. The She2p complex contains the transport machinery that carries the mRNA to its designated place within the cell. 
Table 1 | Pre-rRNAs enriched with Nop15p-PrA

\begin{tabular}{ll}
\hline Gene & rRNA sequences \\
\hline RDN58 & $5.8 \mathrm{~S}$ rRNA \\
ITS2 & ITS2 \\
ETS1 & ETS1 and 35S pre-rRNA \\
RDN25-1 & $25 \mathrm{~S}$ rRNA \\
RDN25-2 & $25 \mathrm{~S}$ rRNA \\
\hline
\end{tabular}

Transcripts listed showed a mean ratio of purified versus total RNA greater than two s.d. from the mean and are listed according to descending mean ranking. All transcripts had been previously identified ${ }^{8}$.

She2p complex, which does not overlap with any of the other isolated mRNPs, represents a late and exclusively cytoplasmic stage of the mRNA pathway, and contains the transport machinery that carries the mRNA to its designated place within the cell ${ }^{28,29}$. By performing six independent isolations of complexes associated with overlapping portions of a common pathway, we verified the specificity of our method in a manner analogous to performing reverse isolations, for example, tagged Sac3p isolates Mex67p, as tagged Mex67p isolates Sac3p, and there are similar examples for the other tagged proteins.

\section{Global analyses of Nop15p and She2p associated RNAs}

We next tested the specificity of the RNAs associated with our isolated RNP complexes. We used microarray analyses to globally examine the RNAs associated with Nop15p and She2p, both of which should be examples of proteins with only a small subset of associating RNAs. The RNAs associated with the protein complexes in each affinity-purification experiment were labeled and hybridized to DNA microarrays that, in the case of Nop15p, also carried probes corresponding to the ribosomal DNA loci. We performed each experiment in triplicate; we defined enriched transcripts as those with enrichment ratios more than two s.d. away from the mean (Supplementary Fig. 2 online).

We found that RNAs that co-isolated with Nop15p-PrA were highly enriched in sequences within the 5.8S, 25S rRNA and the internal transcribed spacer 2 (ITS2; Table 1 and Supplementary Table 3 online). This is consistent with previous findings, which show that Nop15p is primarily associated with precursors containing these same sequences ${ }^{8}$. This also confirms the results above (Fig. 3a), again showing the high degree of specificity in terms of RNAs co-isolating with our RNP complexes.

Our She2p RNP complex isolations form an excellent positive control, as in two recent microarray studies, 11 and 24 transcripts, respectively, were reported to be specifically associated with tagged trimeric She2p-She3p-Myo4p complexes ${ }^{29,30}$. Ten of these RNAs were transcribed from cell cycle-regulated genes, whereas others appeared to participate in pathways related to sensing or responding to stress. The overlap between these previous studies and ours is high: we identified $41 \mathrm{mRNAs}$ that co-isolated with She $2 \mathrm{p}-\mathrm{PrA}$, including $90 \%$ and $65 \%$ of the transcripts previously identified, respectively ${ }^{29,30}$ (Table 2 and Supplementary Table 3). Our results also suggest that the number of transcripts associated with She2p alone is greater than previously reported. We found other cell cycle-regulated transcripts, plus a number of transcripts that point toward additional roles for She $2 p$ in the localization of transcripts to the vacuole, endoplasmic reticulum and mitochondria.

\section{DISCUSSION}

The efficiency of our method does not depend on a particular protein tag. We showed that neither protein nor RNA rearrangements (leading to false positives) are issues during our RNP isolation procedure. Our method is also not limited to use with protein tags; we recently isolated an RNP complex via an RNA tag (data not shown).

We demonstrated the biological relevance of our method by the successful isolation of different pre-ribosomal and mRNAcontaining complexes, some of which have proven difficult candidates for affinity purification in the past because of either low abundance or for other technical reasons ${ }^{1,3,4}$. In every case

Table 2 | mRNAs enriched with She2p-PrA

\begin{tabular}{|c|c|c|}
\hline Gene & Protein localization & References \\
\hline$E R G 2^{\mathrm{a}}$ & ER & 29,30 \\
\hline$P R Y 2^{\mathrm{a}}$ & Vacuole & \\
\hline$A S H 1^{\mathrm{a}}$ & Bud tip & 29,30 \\
\hline$S R L 1^{\mathrm{a}}$ & Vacuole & 29,30 \\
\hline YMRO03W & Mitochondria & \\
\hline YSP2 & Mitochondria & \\
\hline PAC10 & Cytoplasm & \\
\hline$W S C 2^{\mathrm{a}}$ & Bud & 29,30 \\
\hline$M M R 1^{\mathrm{a}}$ & Bud neck, mitochondria & 29,30 \\
\hline$B U D 8^{\mathrm{a}}$ & Bud tip & \\
\hline RHR2 & Cytoplasm & \\
\hline$C L B 2^{\mathrm{a}}$ & Bud neck & 30 \\
\hline NTF2 & Nuclear envelope & \\
\hline YOR248W & Dubious $^{b}$ & 29 \\
\hline RPS11B & Cytoplasm & \\
\hline PMT2 & ER & \\
\hline$A C B 1$ & Cytoplasm & \\
\hline MID2 & Plasma membrane & 30 \\
\hline STM1 & Cytoplasm & \\
\hline IST2 & Bud, plasma membrane & 29,30 \\
\hline SUI1 & Cytoplasm & \\
\hline YLR413W & Unknown & \\
\hline$T C B 2^{\mathrm{a}}$ & Bud & 30 \\
\hline CYS3 & Cytoplasm & \\
\hline CPS1 & Vacuole & \\
\hline$\angle C B 1$ & Plasma membrane & 30 \\
\hline$A D E 3$ & Cytoplasm & \\
\hline$T A M 41^{\mathrm{a}}$ & Bud & 29,30 \\
\hline $\operatorname{IRC} 8^{\mathrm{a}}$ & Bud & 29,30 \\
\hline$T K L 1$ & Cytoplasm & \\
\hline$S C Y 1$ & Vesicles & \\
\hline YEF3 & Cytoplasm & \\
\hline SUN4 & Mitochondria & \\
\hline HOM2 & Cytoplasm & 30 \\
\hline CIS3a & Bud tip & \\
\hline PRY1 & Vacuole & \\
\hline FRS2 & Cytoplasm & \\
\hline MAK21 & Cytoplasm & \\
\hline DED81 & Cytoplasm & \\
\hline TEF4 & Cytoplasm & \\
\hline YLR179C & Cytoplasm & \\
\hline
\end{tabular}

${ }^{a}$ Cell cycle-regulated transcripts. ${ }^{\text {b}} 0$ pen reading frame not confirmed.

Transcripts listed had a mean ratio of purified versus total RNA greater than two s.d. from the mean and are listed according to descending mean ranking. References for previously identified transcripts are given. 
presented here, we characterized many previously unknown interacting proteins associated with these RNP complexes, and for the She2p-associated mRNPs, previously undescribed mRNAs. We also captured components that are known to associate only transiently with RNAs.

Our method is not limited to yeast, and can readily be adapted to other systems, including mammalian-derived ones. Given its ease, wide applicability and high discovery potential, we believe our method is an important addition to the technical repertoire of the biomedical community.

\section{METHODS}

Immunological affinity purification. We thawed frozen cell grindate into an appropriate buffer supplemented with varying amounts of $\mathrm{NaCl}$. We used different amounts of frozen cell grindate for different bait proteins depending on their molecular abundance (Supplementary Fig. 3 online). We clarified cell lysates by filtration. We added washed antibody-conjugated magnetic beads (Dynal) to the cleared cell lysate. The samples were incubated for 5-30 min at $4{ }^{\circ} \mathrm{C}$. We collected the beads using a Dynal magnet, washed them three times with buffer by pipetting and once for $5 \mathrm{~min}$, rotating at room temperature $\left(18-25^{\circ} \mathrm{C}\right)$. We eluted the isolated protein complex from the beads twice for $20 \mathrm{~min}$ at room temperature in fresh aqueous $500 \mathrm{mM} \mathrm{NH}_{4} \mathrm{OH}$, $0.5 \mathrm{mM}$ EDTA solution. We lyophilized the pooled eluates in a SpeedVac (Thermo Savant) overnight, resuspended the pellets in SDS-PAGE sample buffer, separated the samples on a $4-12 \%$ NuPAGE Novex Bis-Tris precast gel (Invitrogen) and visualized them by R250 Coomassie blue staining. Proteins were identified by mass spectrometry (Supplementary Methods).

Additional methods. Detailed descriptions of the above experiments, cell disruption and extraction, and buffer compositions are available in Supplementary Methods. We performed TAP purifications, western blot analyses, RNA quantifications and microarray analyses using published methods as described in Supplementary Methods. Yeast strains used in this work are listed in Supplementary Table 4 online.

Note: Supplementary information is available on the Nature Methods website.

\section{ACKNOWLEDGMENTS}

We thank D. Zenklusen (Albert Einstein College of Medicine) for providing the She2p-PrA and Cbp80p-PrA strains, and F. Stutz (University of Geneva) for providing the antibody to Yra1. We thank all the members of the Rout, Chait and Aitchison laboratories for their support. This work was supported by a grant from the American Cancer Society (RSG0404251) to M.P.R.; by grants from the US National Institutes of Health to B.T.C. (RR00862), J.D.A. (GM067228 and GM076547), and M.P.R., J.D.A. and B.T.C. (RR022220); by a Tri-Institutional Training Fellowship in Chemical Biology to J.A.D.; and by a fellowship from the Charles Revson Foundation to M.0.

\section{Published online at http://www.nature.com/naturemethods/} Reprints and permissions information is available online at http://npg.nature.com/reprintsandpermissions

1. Rigaut, G. et al. A generic protein purification method for protein complex characterization and proteome exploration. Nat. Biotechnol. 17, 1030-1032 (1999).

2. Saveanu, C. et al. Nog2p, a putative GTPase associated with pre-60S subunits and required for late $60 S$ maturation steps. EMBO J. 20, 6475-6484 (2001).
3. Gavin, A.C. et al. Functional organization of the yeast proteome by systematic analysis of protein complexes. Nature 415, 141-147 (2002).

4. Ho, Y. et al. Systematic identification of protein complexes in Saccharomyces cerevisiae by mass spectrometry. Nature 415, 180-183 (2002).

5. Sorger, P.K., Ammerer, G. \& Shore, D. in Protein Function: a Practical Approach. (ed. Creighton, T.) 199-278 (IRL Press, 0xford, 1989).

6. Lewis, J.D., Goerlich, D. \& Mattaj, I. A yeast cap binding protein complex (yCBC) acts at an early step in pre-mRNA splicing. Nucl. Acids Res. 24, 3332-3336 (1996).

7. Gavin, A.C. et al. Proteome survey reveals modularity of the yeast cell machinery. Nature 440, 631-636 (2006).

8. Oeffinger, M. \& Tollervey, D. Yeast Nop15p is an RNA-binding protein required for pre-rRNA processing and cytokinesis. EMBO J. 22, 6573-6583 (2003).

9. Segref, A. et al. Mex67p, a novel factor for nuclear mRNA export, binds to both poly(A)+ RNA and nuclear pores. EMBO J. 16, 3256-3271 (1997).

10. Harnpicharnchai, P. et al. Composition and functional characterization of yeast 66 s ribosome assembly intermediates. Mol. Cell 8, 505-515 (2001).

11. Schafer, T., Strauss, D., Petfalski, E., Tollervey, D. \& Hurt, E. The path from nucleolar $90 \mathrm{~S}$ to cytoplasmic $40 \mathrm{~S}$ pre-ribosomes. EMBO J. 22, 1370-1380 (2003).

12. Henry, Y. et al. The $5^{\prime}$ end of yeast $5.8 \mathrm{~S}$ rRNA is generated by exonucleases from an upstream cleavage site. EMBO J. 13, 2452-2463 (1994).

13. Krogan, N.J. et al. Global landscape of protein complexes in the yeast Saccharomyces cerevisiae. Nature 440, 637-643 (2006).

14. Burckstummer, T. et al. An efficient tandem affinity purification procedure for interaction proteomics in mammalian cells. Nat. Methods 3, 1013-1019 (2006).

15. Hurt, E. et al. Mex67p mediates nuclear export of a variety of RNA polymerase II transcripts. J. Biol. Chem. 275, 8361-8368 (2000).

16. Warner, J.R. The assembly of ribosomes in yeast. J. Biol. Chem. 246, 447-454 (1971).

17. Stewart, M. Ratcheting mRNA out of the nucleus. Mol. Cell 25, 327-330 (2007)

18. Arava, Y. et al. Genome-wide analysis of mRNA translation profiles in Saccharomyces cerevisiae. Proc. Natl. Acad. Sci. USA 100, 3889-3894 (2003).

19. Vinciguerra, P. \& Stutz, F. mRNA export: an assembly line from genes to nuclear pores. Curr. Opin. Cell Biol. 16, 285-292 (2004).

20. Jimeno, S., Rondon, A.G., Luna, R. \& Aguilera, A. The yeast THO complex and mRNA export factors link RNA metabolism with transcription and genome instability. EMBO J. 21, 3526-3535 (2002).

21. Strasser, K. \& Hurt, E. Yra1p, a conserved nuclear RNA-binding protein, interacts directly with Mex67p and is required for mRNA export. EMBO J. 19, 410-420 (2000).

22. Zenklusen, D., Vinciguerra, P., Strahm, Y. \& Stutz, F. The yeast hnRNP-Like proteins Yra1p and Yra2p participate in mRNA export through interaction with Mex67p. Mol. Cell. Biol. 21, 4219-4232 (2001).

23. Vinciguerra, P., Iglesias, N., Camblong, J., Zenklusen, D. \& Stutz, F. Perinuclear Mlp proteins downregulate gene expression in response to a defect in mRNA export. EMBO J. 24, 813-823 (2005).

24. Andrulis, E.D. et al. Esc1, a nuclear periphery protein required for Sir4-based plasmid anchoring and partitioning. Mol. Cell. Biol. 22, 8292-8301 (2002).

25. Duncan, K., Umen, J.G. \& Guthrie, C. A putative ubiquitin ligase required for efficient mRNA export differentially affects hnRNP transport. Curr. Biol. 10, 687-696 (2000).

26. Lei, E.P. et al. Sac3 is an mRNA export factor that localizes to cytoplasmic fibrils of nuclear pore complex. Mol. Biol. Cell 14, 836-847 (2003).

27. Ghaemmaghami, S. et al. Global analysis of protein expression in yeast. Nature 425, 737-741 (2003).

28. Long, R.M. et al. Mating type switching in yeast controlled by asymmetric localization of ASH1 mRNA. Science 277, 383-387 (1997).

29. Takizawa, P.A. \& Vale, R.D. The myosin motor, Myo4p, binds Ash1 mRNA via the adapter protein, She3p. Proc. Natl. Acad. Sci. USA 97, 5273-5278 (2000).

30. Shepard, K.A. et al. Widespread cytoplasmic mRNA transport in yeast: identification of 22 bud-localized transcripts using DNA microarray analysis. Proc. Natl. Acad. Sci. USA 100, 11429-11434 (2003).

31. Reck-Peterson, S.L., Tyska, M.J., Novick, P.J. \& Mooseker, M.S. The yeast class V myosins, Myo2p and Myo4p, are nonprocessive actin-based motors. J. Cell Biol. 153, 1121-1126 (2001).

32. Pruyne, D. \& Bretscher, A. Polarization of cell growth in yeast. J. Cell Sci. 113, 571-585 (2000).

33. Rout, M.P. et al. The yeast nuclear pore complex: composition, architecture, and transport mechanism. J. Cell Biol. 148, 635-651 (2000). 